Editorial

\title{
Acknowledgment to Reviewers of Pollutants in 2021
}

\section{Pollutants Editorial Office}

Citation: Pollutants Editorial Office.

Acknowledgment to Reviewers of Pollutants in 2021. Pollutants 2022, 2, 21-22. https://doi.org/10.3390/ pollutants2010003

Published: 27 January 2022

Publisher's Note: MDPI stays neutral with regard to jurisdictional claims in published maps and institutional affiliations.

Copyright: () 2022 by the author. Licensee MDPI, Basel, Switzerland. This article is an open access article distributed under the terms and conditions of the Creative Commons Attribution (CC BY) license (https://creativecommons.org/licenses/by/4.0/).

MDPI AG, St. Alban-Anlage 66, 4052 Basel, Switzerland

Rigorous peer-reviews are the basis of high-quality academic publishing. Thanks to the great efforts of our reviewers, Pollutants was able to maintain its standards for the high quality of its published papers. Thanks to the contribution of our reviewers, in 2021, the median time to first decision was 35 days and the median time to publication was 63 days. The editors would like to extend their gratitude and recognition to the following reviewers for their precious time and dedication, regardless of whether the papers they reviewed were finally published:

\begin{tabular}{|c|c|}
\hline Ahmed Eid & Maria Toscanesi \\
\hline Ahmed Saud Abdulhameed & Marina Lasagni \\
\hline Anatolii Kucher & Marinela Inta \\
\hline Anna Budka & Martina Grifoni \\
\hline Antonio Satriani & Massimo Trotta \\
\hline Boubié Guel & Mats Leifels \\
\hline Brett Singer & Mauro Marini \\
\hline Chen $\mathrm{Xu}$ & Mohammad Shahid \\
\hline Chen-Yi Sun & Mostafa Redwan \\
\hline Concetta Pironti & Mouheb Chebbi \\
\hline Dario Savoca & Murthy Chavali \\
\hline Dawn Ford & Nur Hashimah Alias \\
\hline Debojyoti Moulick & Obdulia Monteserín-Abella \\
\hline Deepika Malhotra & Omid Aminoroayaie Yamini \\
\hline Dmitry Vlasov & Orhan Baytar \\
\hline Douglas Collins & Osmel La-Llave-Leon \\
\hline Efthimios Zervas & Pierre Herckes \\
\hline Elena Koumaki & Pierre-Yves Pascal \\
\hline Elena Matei & Prateek M. Shrestha \\
\hline Elsayed Haggag & Raimundo Jiménez Ballesta \\
\hline Eun-Hee Koh & Rajender Gupta \\
\hline Flávio Manoel Rodrigues Da Silva-Júnior & Ramona Zgavarogea \\
\hline Francesco Saliu & Rui Hou \\
\hline Ilaria Guagliardi & Ruilian Zhang \\
\hline Joanna Jaskula & Santiago Mejía-Dugand \\
\hline José Herrera-Melián & Sergey Vinokurov \\
\hline Joseph Colosi & Sergio Santana-Viera \\
\hline Junjie Zhang & Shaojie Song \\
\hline Luca Burratti & Stuart Batterman \\
\hline Luís Pinho & Taghi Miri \\
\hline Manuel Arias Ballesteros & Tanvir Khan \\
\hline Margarita Bakaeva & Valérie Simon \\
\hline Maria Giaoutzi & Xuechao Gao \\
\hline Maria Ricciardi & Xueyang Zhang \\
\hline
\end{tabular}

Ahmed Eid

Ahmed Saud Abdulhameed

Anatolii Kucher

Anna Budka

Antonio Satriani

Boubié Guel

Brett Singer

Chen Xu

Chen-Yi Sun

Concetta Pironti

Debojyoti Moulick

Deepika Malhotra

Elena Koumaki

Elena Matei

Elsayed Haggag

Eun-Hee Koh

Flávio Manoel Rodrigues Da Silva-Júnior

Francesco Saliu

Ilaria Guagliardi

Joanna Jaskula

José Herrera-Melián

Joseph Colosi

Junjie Zhang

Luca Burratti

uís Pinho

Manuel Arias Ballesteros

Maria Giaoutzi

\author{
Maria Toscanesi \\ Marina Lasagni \\ Marinela Inta \\ Martina Grifoni \\ Massimo Trotta \\ Mats Leifels \\ Mauro Marini \\ Mohammad Shahid \\ Mostafa Redwan \\ Nur Hashimah Alias \\ Omid Aminoroayaie Yamini \\ n Baytar \\ ( \\ rre Herckes \\ Pierre-Yves Pascal \\ Prateek M. Shrestha \\ Raimundo Jiménez Ballesta \\ Rajender Gupta \\ Ramona Zgavarogea \\ Rui Hou \\ Ruilian Zhang \\ Santiago Mejía-Dugand \\ Sergey Vinokurov \\ Sergio Santana-Viera \\ Shaojie Song \\ Stuart Batterman \\ Taghi Miri \\ Xuechao Gao \\ Xueyang Zhang
}


Ylenia Miele

Zongbo Shi

Zineb Faiz 